\title{
Evaluación del crecimiento y producción de biomasa de dos cepas del género Pleurotus spp., cultivadas en un medio agar con diferentes sustratos
}

\section{Evaluation of growth and biomass production of two strains of the Pleurotus spp., cultivated in an agar medium with different substrates}

\author{
${ }^{\bullet}$ Carol Daniela Coello-Loor ${ }^{1}$, Juan Humberto Avellaneda-Cevallos ${ }^{2}$, Alexandra Elizabeth Barrera-Álvarez ${ }^{2}$, \\ Mayra Mercedes Peña-Galeas ${ }^{3}$, Piedad Francisca Yépez Macías², Elizabeth Ruth Racines-Macías² \\ ${ }^{1}$ Universidad Técnica Estatal de Quevedo. Facultad de Ciencias Pecuarias. Carrera de Ingeniería en Alimentos. \\ Campus Finca Experimental "La María”. CP. 121250. Km. 7 1/2 vía El Empalme, cantón Mocache. \\ Los Ríos. Ecuador. ${ }^{\bullet}$ ccoello@uteq.edu.ec \\ ${ }^{2}$ Universidad Técnica Estatal de Quevedo. Facultad de Ciencias Pecuarias. Carrera de Ingeniería Zootécnica. Campus Finca \\ Experimental “La María”. CP. CP. 121250. Km. 7 1/2 vía El Empalme, cantón Mocache, Los Ríos. Ecuador. \\ javellaneda@uteq.edu.ec; abarrera@uteq.edu.ec; pyepez@uteq.edu.ec; eracines@uteq.edu.ec \\ ${ }^{3}$ Instituto Nacional de Investigaciones Agropecuarias-INIAP. Estación Experimental Tropical Pichilingue. CP. 121250. \\ Km 5 1/2 vía Quevedo-El Empalme, cantón Mocache, Los Ríos. Ecuador. mayra.pena.galeas@gmail.com
}

Rec.: 10.04.2017. Acept.: 28.08.2017. Publicado el 1 de diciembre de 2017

Resumen

$\mathrm{L}$ a velocidad de crecimiento radial (VCR) $\left(\mathrm{mm} \cdot \mathrm{h}^{-1}\right)$ y la producción de biomasa (PB) (g.g ${ }^{-1}$ de sustrato seco) son técnicas que puedan establecer el grado de adaptación y desarrollo de los hongos del género Pleurotus spp., a distintos sustratos que podrían emplearse en una fermentación en medio sólido. Las especies fueron Pleurotus sapidus (Ps) y Pleurotus ostreatus IE8 (Po). El medio de cultivo sintético empleado fue el papa dextrosa agar (PDA), con un $\mathrm{pH}$ que va de 5.6 a 5.9, ideal para el crecimiento de hongos, tiene todos los componentes nutritivos, y ligera acidez que logran la inhibición del desarrollo de bacterias; diluido en 4 diferentes soluciones preparadas con los materiales residuales (solución cascarilla de arroz CaPDA, solución cáscara de maracuyá CmPDA, solución mezcla 50\% Cascarilla de arroz $+50 \%$ Cáscara de maracuyá CaCmPDA y agua destilada+PDA) con el propósito de observar el crecimiento radial cada 24 horas y la producción de biomasa fúngica de estos hongos lignocelulósicos por su periodo de incubación y la adaptación a nivel in vitro. Los tratamientos que presentaron mejor comportamiento VCR fueron el PoCaPDA (0.569) y el PoCaCmPDA (0.549); la cepa que reporto valores más altos en VCR y PB fue el Pleurotus ostreatus y el mejor medio de cultivo fue el CaPDA, en ambas variables; mientras, la mayor producción de biomasa fue en Pleurotus sapidus en CaPDA (0.1727) y el Pleurotus ostreatus IE8 en CmPDA (0.1722), CaPDA (0.1706) y PDA (0.1694).

Palabras clave: Hongos lignocelulósicos, cascara de maracuyá, cascarilla de arroz, papa dextrosa agar, crecimiento radial.
The rate of radial growth VCR $\left(\mathrm{mm} \cdot \mathrm{h}^{-1}\right)$ and biomass 1 production PB (g.g ${ }^{-1}$ of dry substrate) are techniques that help us to understand the degree of adaptation and development of mushrooms of the Pleurotus spp., genus in various substrates which could be applied in a solid medium fermentation. The synthetic culture medium used was potato dextrose agar (PDA), with a $\mathrm{pH}$ of $5.6 \mathrm{q} 5.9$ is ideal for fungal growth, as it has all the nutritional components and due to its slight acidity helps to inhibition the growth of bacteria, diluted in 4 different solutions prepared with the residual materials, with the purpose to observe the radial growth every 24 hours and the fungal biomass production of these lignocellulosic mushrooms during their period of incubation and adaptation which they could have in vitro. The species were Pleurotus sapidus and Pleurotus ostreatus IE8. the best VCR showed the treatments PoCaPDA and PoCaCmPDA and the better strain was the Pleurotus ostreatus in VCR and PB and the best culture medium was the CaPDA in both variables. Whereas in the production biomass treatments showed better results adaptation was the CaPDA Pleurotus sapidus (0.1727) and with CmPDA IE8 Pleurotus ostreatus (0.1722) CaPDA (0.1706) and PDA (0.1694).

Key words: Lignocellulosic fungi, passion fruit husk, rice husk, potato dextrose agar, radial growth. 


\section{Introducción}

$\mathrm{L}$ producción de hongos representa una alternativa de bioconversión para el desarrollo de nuevas áreas de producción, con un impacto benéfico en el crecimiento económico debido a que incorpora la reutilización de desechos ricos en lignocelulosa, material que representa cerca del $40 \%$ de la biomasa producida por la fotosíntesis y que no puede ser aprovechada en forma directa para la alimentación humana y animal, debido a su baja digestión (Arias-Carbajal et al., 2005; Martinez et al., 2015).

El uso del cultivo de Pleurotus spp., en diferentes residuos agroindustriales permite impulsar el abasto alimentario, la generación de ingresos económicos y el cuidado del medio ambiente, al evitar la acumulación o quema de estos residuos en las comunidades rurales (PinedaInsuasti et al., 2013; Gaitán-Hernández y Silva-Huerta, 2016).

El cultivo de los hongos del género Pleurotus spp. es de importancia debido a que producen proteínas de alta calidad sobre un sustrato que consiste en materiales de desecho de carácter lignocelulósico. La capacidad de los hongos para adaptar rápidamente su metabolismo a diversas fuentes de carbono y nitrógeno, depende de la habilidad del hongo para la formación de micelio y permitir su desarrollo. La velocidad de crecimiento radial (VCR) y la producción de biomasa (PB) son métodos que nos permiten evaluar el comportamiento de estos hongos en diferentes condiciones en el medio de cultivo (Rojas y Hormaza, 2014).

La cascarilla de arroz es el elemento que acompaña a dicho grano hasta antes del proceso de secado y descascarillado. Generalmente, la cascarilla se trasporta al basurero o se quema sin aprovechar la energía generada. La cascarilla de arroz o tamo corresponde al $22 \%$ en un proceso de pilado de arroz. La cascarilla tiene un aspecto parecido a la paja, es muy ligera. Uno de los elementos que requieren los hongos del género Pleurotus spp., es la celulosa (C6H10O5)n, siendo el componente principal de las fibras de este subproducto agrícola (Valverde et al., 2007).

En la industria de concentrados de maracuyá, se tienen aproximadamente 25,945 toneladas de desecho. Actualmente, una pequeña porción de los desechos se utiliza para la alimentación de animales, pero se podrían obtener varios subproductos que darían mayores ingresos a la industria de concentrados. Las cáscaras que corresponden al $50-60 \%$ del peso total de la fruta, constituye el desecho más molesto en las procesadoras, a pesar de ser una buena fuente de proteína, pectina y minerales. Un análisis de la cáscara de maracuyá deshidratada reportó que tiene $16.80 \%$ de humedad, $4.58 \%$ de proteína cruda, $0.33 \%$ de extracto etéreo, $6.76 \%$ de cenizas totales, $25.66 \%$ de fibra cruda y el $45.87 \%$ de extracto libre de nitrógeno (Cuesta y Muñoz, 2010; Cruz et al., 2015).
Por consiguiente, conviene desarrollar un proceso orientado al aprovechamiento integral de recursos provenientes de la producción agroindustrial, donde, reduciría la contaminación ambiental por los residuos derivados de estas actividades. Entre estos residuos está la cáscara de maracuyá y la cascarilla de arroz, que, por sus volúmenes, resulta atractivos para la aplicación de esta biotecnología, por tanto, el presente trabajo evaluó la velocidad de crecimiento radial y producción de biomasa de dos cepas del género Pleurotus spp., con el fin de demostrar que el hongo podría adaptarse a las características de los dos sustratos (cáscara de maracuyá y cascarilla de arroz).

\section{Materiales y métodos}

T a investigación se desarrolló en el Laboratorio de Rumiología y Metabolismo Nutricional "RUMEN" de la Universidad Técnica Estatal de Quevedo. Se aplicó un diseño completamente al azar con arreglo factorial siendo los factores de estudio dos Cepa de Pleurotus (Pleurotus sapidus y Pleurotus ostreatus) y Medio de cultivo PDA (preparados en solución de cascarilla de arroz, Solución de cáscara de maracuyá, Mezcla solución cascarilla de arroz $(50 \%)+$ solución cáscara de maracuyá $(50 \%)$ y agua destilada), dando como producto ocho tratamientos, con ocho repeticiones para el estudio de la velocidad de crecimiento radial y tres para la producción de biomasa (Cuadro 1).

\section{Preparación de los medios de cultivo}

Se colocó en cada vaso de precipitación $100 \mathrm{~g}$ de cascarilla de arroz lavada, 100 g de cáscara de maracuyá picada y lavada, $50 \mathrm{~g}$ de cáscara de maracuyá picada y lavada $+50 \mathrm{~g}$ de cascarilla de arroz lavada, con un litro de agua destilada. Se llevó al fuego y se dejó hervir por 30 minutos. Se tamizó cada preparación. Se diluyó $39 \mathrm{~g}$ de PDA en cada solución preparada. En un litro de agua destilada se disolvió $39 \mathrm{~g}$ de PDA para el último medio de cultivo. Se calentaron las muestras de los medios de cultivo para diluir uniformemente el agar en la preparación, hasta que hiervan por un minuto. Se esterilizaron en autoclave a $121^{\circ} \mathrm{C}$ durante 15 minutos. Se llevó al baño maría a $50{ }^{\circ} \mathrm{C}$ una vez salido de la autoclave. En total se obtuvieron cuatro medios de cultivo: CaPDA (cascarilla de arroz + PDA), CmPDA (cáscara de maracuyá + PDA), CaCmPDA (cascarilla de arroz 50\%+ cáscara de maracuyá 50\% + PDA), PDA (agua destilada + PDA).

\section{Determinación de curva de crecimiento micelial}

Se inoculó con implantes de micelio activo de $4 \mathrm{~mm}$ de diámetro en el centro de una caja petri de $9 \mathrm{~cm}$, con $15 \mathrm{~mL}$ de medio de cultivo, se incubó a $28^{\circ} \mathrm{C}$ y se realizó con el uso de un vernier, mediciones diarias del diámetro de crecimiento 
Cuadro 1. Composición de los tratamientos experimentales

\begin{tabular}{cclll}
\hline \multicolumn{4}{c}{ Tratamientos } & \\
\cline { 1 - 3 } $\mathrm{N}^{\circ}$ & \multicolumn{2}{c}{ Símbolo Código } & \multicolumn{1}{c}{ Detalles } \\
\hline $\mathrm{T}_{1}$ & $\mathrm{a}_{1} \mathrm{~b}_{1}$ & PsCaPDA & P. sapidus + solución cascarilla de arroz \\
$\mathrm{T}_{2}$ & $\mathrm{a}_{1} \mathrm{~b}_{2}$ & PsCmPDA & P. sapidus + solución cáscara de maracuyá \\
$\mathrm{T}_{3}$ & $\mathrm{a}_{1} \mathrm{~b}_{3}$ & PsCaCmPDA & P. sapidus + solución mezcla $\mathrm{b}_{1}(50 \%)+\mathrm{b}_{2}(50 \%)$ \\
$\mathrm{T}_{4}$ & $\mathrm{a}_{1} \mathrm{~b}_{4}$ & PsPDA & P. sapidus + agua destilada \\
$\mathrm{T}_{5}$ & $\mathrm{a}_{2} \mathrm{~b}_{1}$ & PoCaPDA & P. ostreatus + solución cascarilla de arroz \\
$\mathrm{T}_{6}$ & $\mathrm{a}_{2} \mathrm{~b}_{2}$ & PoCmPDA & P. ostreatus + solución cáscara de maracuyá \\
$\mathrm{T}_{7}$ & $\mathrm{a}_{2} \mathrm{~b}_{3}$ & PoCaCmPDA & P. ostreatus + solución mezcla $\mathrm{b}_{1}(50 \%)+\mathrm{b}_{2}(50 \%)$ \\
$\mathrm{T}_{8}$ & $\mathrm{a}_{2} \mathrm{~b}_{4}$ & PoPDA & P. ostreatus + agua destilada \\
\hline
\end{tabular}

$\mathrm{b}_{1}=$ cascarilla de arroz; $\mathrm{b}_{2}=$ cáscara de maracuyá

del micelio de las dos cepas del género Pleurotus spp., hasta la invasión total de la caja petri. La tasa de crecimiento específico es el valor que se obtiene de realizar un análisis de regresión lineal de cada tratamiento para conocer la velocidad de crecimiento radial que tienen los hongos del género Pleurotus spp.

\section{Determinación de biomasa}

Se colocaron 40 papeles filtro en la estufa a $60{ }^{\circ} \mathrm{C}$ por 48 horas, se pasaron al desecador por $30 \mathrm{~min}$, se pesaron y se rotularon. Para la determinación de la biomasa se utilizaron 2 métodos detallados a continuación:

Método 1: Se agregó el contenido de una caja de agar con micelio en un vaso de precipitación con $200 \mathrm{~mL}$ de agua destilada, se llevó al microondas hasta que se disuelva el agar, se pasó por el papel filtro, con la ayuda de una bomba al vacío, esto agilizó el proceso de filtrado. Se lavó con agua destilada caliente, para disolver completamente el residuo de agar. Se llevó cada papel a la estufa a $60^{\circ} \mathrm{C}$ por 48 horas y posteriormente al desecador por 30 minutos, se tomaron los pesos con la balanza analítica (Peláez-Acero et al., 2011).

Método 2: Se hizo la determinación de biomasa del micelio crecido en medio sólido, agregando el contenido de una caja de agar con micelio en un matraz de $250 \mathrm{~mL}$, posteriormente se cubrió el matraz con aluminio, se congeló por 12 horas, transcurrido ese tiempo se agregó $125 \mathrm{~mL}$ de agua destilada, se esterilizó en autoclave a $121{ }^{\circ} \mathrm{C}$ y 15 libras de presión durante 20 minutos, se filtró en caliente y el papel filtro con la muestra se llevó a la estufa a $60{ }^{\circ} \mathrm{C}$ por 48 horas, seguido se ubicó en el desecador por 30 minutos. Se pesaron las muestras y se determinó la biomasa por diferencia de peso (Peláez-Acero et al., 2011).

\section{Resultados y discusión}

$\mathrm{L}$ a invasión total del micelio en las cajas petri fue a las 192 horas; se observó que por efecto de la cepa (Cuadro 2) a las 48 y 72 horas de incubación existió diferencia ( $<<.0001$ ), así, la cepa del P. sapidus fue la mejor con 9.4063 $\mathrm{mm}$ y $18.2344 \mathrm{~mm}$, respectivamente, panorama que cambia notablemente a partir de las 120 hasta las 192 horas, donde la cepa del $P$. ostreatus se destacó con mayor diámetro de crecimiento, con valores desde $44.031 \mathrm{~mm}$ hasta $83.625 \mathrm{~mm}$.

Este comportamiento demuestra que la cepa que tuvo mayor habilidad para adaptarse rápidamente en el medio del cultivo fue la cepa $P$. sapidus, sin embargo, la cepa $P$. ostreatus alcanzó una velocidad de crecimiento radial mayor a partir de las 120 horas. En cuanto a la variable de biomasa fúngica ( $\mathrm{p}=0.0003$ ), de mejor comportamiento, fue la cepa $P$. ostreatus con una media de $0.165042 \mathrm{~g} \mathrm{~g}^{-1}$ de sustrato seco.

El mejor medio para el crecimiento radial $(\mathrm{p}<.0001)$, en todas las horas de incubación, fue el PDA diluido en solución de cascarilla de arroz CaPDA (Cuadro 3), que manifestó una rápida adaptación y mejoró el proceso de crecimiento, no obstante, los reportes por Rios et al. (2010) y, Suarez y Holguin (2011) indican que tardan 9 y 10 días, respectivamente, en invadir por completo la caja petri, utilizando el PDA comercial diluido en agua destilada. En cuanto a la mayor producción de biomasa fúngica, el medio de cultivo CaPDA, reportó valores de $0.171617 \mathrm{~g} \mathrm{~g}^{-1}$ de sustrato seco $(\mathrm{p}<.0001)$. Una de las razones, son los componentes de la cascarilla de arroz, con aproximadamente, 29 al 35\% de celulosa, 18 al 22\% de hemicelulosa y 18 al $25 \%$ de lignina, en su estructura (Valverde et al., 2007), además, los Pleurotus oxidan el polímero de lignina por el contenido de enzimas como las lacasas, xilanasas, lignina peroxidasas, manganeso 
Cuadro 2. Efecto de las cepas del género Pleurotus spp., en la velocidad de crecimiento radial y en la producción de biomasa $(\mathrm{mm})$

\begin{tabular}{|c|c|c|c|c|}
\hline \multirow{2}{*}{ Horas de incubación } & \multicolumn{2}{|c|}{ Cepas } & \multirow{2}{*}{$\Sigma \Sigma M$} & \multirow{2}{*}{$\mathbf{P}<$} \\
\hline & Pleurotus sapidus & Pleurotus ostreatus & & \\
\hline 24 & $1.0156 \mathrm{a}$ & $1.0000 \mathrm{a}$ & 0.0320 & 0.7318 \\
\hline 48 & $9.4063 \mathrm{a}$ & $7.7969 \mathrm{~b}$ & 0.1216 & $<.0001$ \\
\hline 72 & $18.2344 \mathrm{a}$ & $16.4531 \mathrm{~b}$ & 0.2190 & $<.0001$ \\
\hline 96 & $28.5156 \mathrm{a}$ & $27.7813 \mathrm{a}$ & 0.3218 & 0.1122 \\
\hline 120 & $40.8594 \mathrm{~b}$ & $44.0313 \mathrm{a}$ & 0.4542 & $<.0001$ \\
\hline 144 & $54.3438 \mathrm{~b}$ & $60.0625 \mathrm{a}$ & 0.3644 & $<.0001$ \\
\hline 168 & $65.0156 \mathrm{~b}$ & $72.3125 \mathrm{a}$ & 0.3766 & $<.0001$ \\
\hline 192 & $75.7688 \mathrm{~b}$ & $83.6250 \mathrm{a}$ & 0.3713 & $<.0001$ \\
\hline $\begin{array}{l}\text { Biomasa } \text { g g}^{-1} \text { de } \\
\text { sustrato seco }\end{array}$ & $0.1513 \mathrm{~b}$ & $0.1650 \mathrm{a}$ & 0.0021 & 0.0003 \\
\hline
\end{tabular}

Medias con una letra común, no son significativamente diferentes (Tukey, p>0.05)

peroxidasas, entre otras; desdoblan la celulosa y la hemicelulosa en pequeñas subunidades monómeras a medida que crecen y se desarrollan sobre los sustratos (Peláez-Acero et al., 2011).

Por otro lado, en la interacción de los factores (cepas y medio de cultivo), el PsCaPDA fue mayor $(\mathrm{p}<.0001)$ a las 24 horas en velocidad de crecimiento radial, con una media de 1.375 mm; mientras, el PsCaCmPDA y el PsCaPDA fueron similares a las 48 horas $(\mathrm{p}>0.05)$ con $10.750 \mathrm{~mm}$ y 10.875 $\mathrm{mm}$, respectivamente, sin embargo, fueron diferentes en comparación con los demás tratamientos $(\mathrm{p}=0.0362)$, con mayor crecimiento micelial.
A partir de las 144 horas de incubación el PoCaPDA en relación con el PsCaPDA difiere significativamente $(p=0.1229)$ con el mismo medio de cultivo y bajo las mismas condiciones de incubación, debido a la diferencias de capacidad de adaptación de las cepas del genero Pleurotus spp. (Cuadro 4), es decir la cepa del P. ostreatus pasó a ser la cepa más veloz en el mismo medio de crecimiento CaPDA. Esto es posible por diferentes causas, una de ellas, es que el $P$. ostreatus tardó más tiempo en adaptarse a las condiciones fisicoquímicas y nutritivas del medio de cultivo, o que el $P$. sapidus al crecer más rápido, agotó las fuentes de carbono y nitrógeno necesarias para su desarrollo, por tanto, su

Cuadro 3. Efecto del medio de cultivo en la velocidad de crecimiento radial y en la producción de biomasa

\begin{tabular}{|c|c|c|c|c|c|c|}
\hline \multirow{2}{*}{$\begin{array}{l}\text { Horas de } \\
\text { Incubación }\end{array}$} & \multicolumn{4}{|c|}{ Medio de Cultivo } & \multirow{2}{*}{$\Sigma \Sigma M$} & \multirow{2}{*}{$\mathbf{P}<$} \\
\hline & CaPDA & CmPDA & CaCmPDA & PDA & & \\
\hline 24 & $1.1875 \mathrm{a}$ & $0.8437 \mathrm{~b}$ & $1.0000 \mathrm{~b}$ & $1.0000 \mathrm{~b}$ & 0.0020 & $<.0001$ \\
\hline 48 & $9.8125 \mathrm{a}$ & $8.7188 \mathrm{~b}$ & $9.6875 \mathrm{a}$ & $6.1875 \mathrm{c}$ & 0.0296 & $<.0001$ \\
\hline 72 & $20.9688 \mathrm{a}$ & $15.0625 \mathrm{c}$ & $19.2188 \mathrm{~b}$ & $14.1250 \mathrm{c}$ & 0.0959 & $<.0001$ \\
\hline 96 & $33.5625 \mathrm{a}$ & $25.4063 \mathrm{c}$ & $30.2500 \mathrm{~b}$ & $23.3750 \mathrm{~d}$ & 0.2071 & $<.0001$ \\
\hline 120 & $50.7500 \mathrm{a}$ & $38.5000 \mathrm{c}$ & $46.1875 \mathrm{~b}$ & $34.3438 \mathrm{~d}$ & 0.4126 & $<.0001$ \\
\hline 144 & $65.6875 \mathrm{a}$ & $52.7188 \mathrm{c}$ & $63.3125 \mathrm{~b}$ & $47.0938 \mathrm{~d}$ & 0.2656 & $<.0001$ \\
\hline 168 & $77.4375 \mathrm{a}$ & $64.3750 \mathrm{c}$ & $74.9375 \mathrm{~b}$ & $57.9063 \mathrm{~d}$ & 0.2837 & $<.0001$ \\
\hline 192 & $87.5313 \mathrm{a}$ & $75.7188 \mathrm{c}$ & $85.3125 \mathrm{~b}$ & $70.2250 \mathrm{~d}$ & 0.2757 & $<.0001$ \\
\hline $\begin{array}{l}\text { Biomasa } \mathrm{g} \mathrm{g}^{-1} \\
\text { de sustrato seco }\end{array}$ & $0.1716 \mathrm{a}$ & $0.1630 \mathrm{ab}$ & $0.1464 \mathrm{c}$ & $0.1516 \mathrm{bc}$ & 0.0029 & $<.0001$ \\
\hline
\end{tabular}

Medias con una letra común, no son significativamente diferentes (Tukey, p>0.05) 


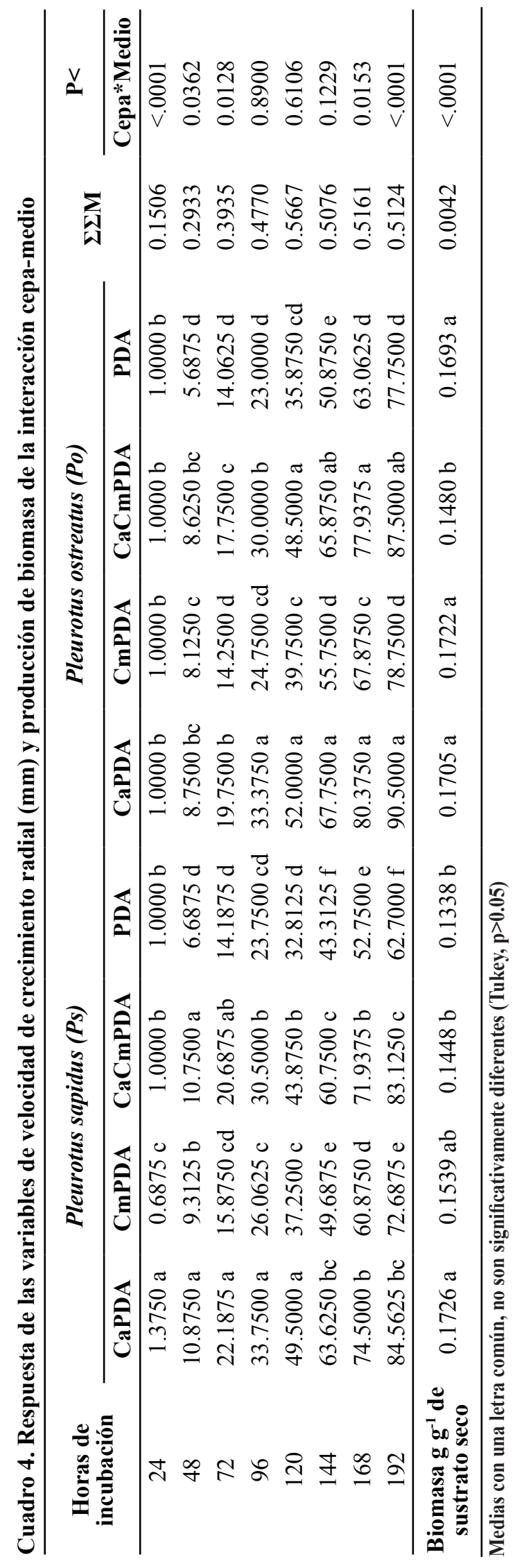


crecimiento pasadas las 96 horas fue más lento, basados en la velocidad de crecimiento radial; método que permite evaluar la capacidad de los hongos lignocelulósicos del género Pleurotus spp., en adaptar su metabolismo a diversas fuentes de carbono y nitrógenos e invadir el sustrato.

Al término de la medición, los tratamientos con mayor crecimiento radial, fue el $P$. ostreatus con los medios de cultivo CaPDA y CaCmPDA con $90.50 \mathrm{~mm}$ y $87.5 \mathrm{~mm}$, respectivamente $(\mathrm{p}<.0001)$, comportamiento posible a los medios de cultivo, que le entregaron al hongo el sustrato lignocelulosico, que sustentó su mayor desarrollo, respuesta similar a la reportada por Peláez-Acero et al. (2011).

En el efecto de la interacción cepa-medio, con respecto a la producción de biomasa (Cuadro 4), los valores más altos $(\mathrm{p}=0.0018)$ se encontraron en el $P$. sapidus en medio CaPDA con $0.1726, P$. ostreatus en medio CmPDA con 0.1722, $P$. ostreatus en medio CaPDA con 0.1705 g.g ${ }^{-1}$ de sustrato seco, respectivamente. Mientras, Peláez-Acero et al. (2011) reportaron 0.193 y $0.13 \mathrm{~g} . \mathrm{g}^{-1}$ de sustrato seco para $P$. ostreatus y $P$. sapidus, correspondientemente, valor superior e inferior a los reportados en esta investigación; esto ocurre a las diferencias que existen en las características físico-químicas y nutricionales del bagazo de caña, cascarilla de arroz y cáscara de maracuyá.

La tasa de crecimiento específico permite conocer la velocidad de crecimiento radial que tienen los hongos del género Pleurotus, y se obtiene del análisis de regresión lineal de cada tratamiento (Martinez et al., 2015). En este caso, se observó que la cepa $P$. ostreatus tuvo mayor velocidad de crecimiento radial con el medio de cultivo CaPDA y CaCmPDA (0.569 $\mathrm{mm} \mathrm{h}^{-1}$ y $\left.0.549 \mathrm{~mm} \mathrm{~h}^{-1}\right)$, respectivamente; situación que se asemeja al uso de la cepa $P$. sapidus en los mismos medios de cultivo (Cuadro 5).

Sanchez et al. (2008) estudiaron el comportamiento de crecimiento de la cepa $P$. ostreatus a $28{ }^{\circ} \mathrm{C}$, en un medio de cultivo agar elaborado a partir de la mezcla de dos residuos lignocelulosicos (rastrojo de tomate y paja de trigo). Midieron el crecimiento radial cada dos dias, reportando un total de 9 dias para la invasión en la caja petri, valores superiores a los reportados en esta investigacion ( 8 dias), lo que indica que la velocidad de crecimiento del $P$. ostreatus fue superior en funcion del tiempo de incubacion.

Sin embargo, Peláez-Acero et al. (2011) obtuvieron valores superiores, al utilizar extracto de bagazo de caña con el P. ostreatus IE8, mejorando el aprovechamiento lignocelulósico a una velocidad de crecimiento radial (VCR) de $0.647 \mathrm{~mm} \cdot \mathrm{h}^{-1}$ y $0.573 \mathrm{~mm} \cdot \mathrm{h}^{-1}$ para $P$. sapidus. Asimismo, Martinez et al. (2015) reportaron VCR de 0.777 $\mathrm{mm} \mathrm{h}^{-1}, 1.26 \mathrm{~mm} \mathrm{~h}^{-1}$ y $1.10 \mathrm{~mm} \mathrm{~h}^{-1}$, para diferentes cepas de $P$. ostreatus cultivadas en medio de agar papa glucosado; valores superiores a los reportados en esta investigaciónm, debiéndose a las características lignocelulosicas de cada residuo o por las fuentes de carbono y nitrógeno de los medios de cultivo utilizados. Por otro lado, Suarez y Holguin (2011), presentaron una invasión de micelio de $45 \mathrm{~mm}$ en 10 días, una VCR de $0.1875 \mathrm{~mm} \mathrm{~h}^{-1}$ con $P$. ostreatus sobre el medio comercial papa dextrosa agar (PDA). Por consiguiente, Rios et al. (2010) obtuvieron una VCR de $0.3 \mathrm{~mm} \mathrm{~h}^{-1}$ con $P$. ostreatus en medio PDA, valores inferiores para los obtenidos en este trabajo.

Bernardi et al. (2013), realizaron una invasión de micelio del hongo de genero $P$. ostreatus en medio de cultivo CDA (pasto elefante+dextrosa+agar), a $28{ }^{\circ} \mathrm{C}$ colonizando la caja Petri en su totalidad a los 10 días de incubacion, posteriormente utilizaron cuatro sustratos diferentes, entre ellos la cascarilla de arroz, donde reportaron una VCR de $0.883 \mathrm{~cm} \mathrm{~h}^{-1}$ en $P$. ostreatus durante 15 dias de incubación, equivalente a $0.3086 \mathrm{~mm} \cdot \mathrm{h}^{-1}$, valores inferiores a los reportados en esta investigación. Sánchez-Santillán et al. (2015), evaluaron velocidad de crecimiento radial al $P$. ostreatus IE8, en medio agar con extracto de bagazo de caña con y sin peptona de caseína, reportando valores de 0.406 y $0.616 \mathrm{~mm} . \mathrm{h}^{-1}$, respectivamente; valores inferiores a los presentados en esta investigación al adicionar peptona de caseina, pero superiores con respecto al medio elaborado sin peptona de caseina. Wisbeck et al. (2016), incubaron cepas del hongo $P$. ostreatus en extracto de trigo y hojas de palma de durazno, para medir su velocidad de crecimiento radial, reportando 8.87 y $6.63 \mathrm{~mm} \cdot \mathrm{dia}^{-1}$, respectivamente. Estos valores son inferiores a los reportados en esta investigación, considerando que la VCR en función de $\mathrm{mm} \cdot \mathrm{h}^{-1}$ sería de 0.369 para $P$. ostreatus en extracto de trigo y 0.276 en

Cuadro 5. Ecuaciones de crecimiento de los hongos del género Pleurotus spp., obtenidas del análisis matemático de regresión lineal de cada tratamiento

\begin{tabular}{lcccc}
\hline \multirow{2}{*}{ Medio de cultivo } & \multicolumn{4}{c}{ Hongo Pleurotus } \\
\cline { 2 - 5 } & Pleurotus sapidus & $\mathbf{R}^{\mathbf{2}}$ & Pleurotus ostreatus & $\mathbf{R}^{\mathbf{2}}$ \\
\hline CAPDA & $\mathrm{Y}=0.516 \mathrm{x}-13.19$ & 0.996 & $\mathrm{Y}=0.569 \mathrm{x}-17.27$ & 0.991 \\
CMPDA & $\mathrm{Y}=0.433 \mathrm{x}-12.79$ & 0.992 & $\mathrm{Y}=0.487 \mathrm{x}-16.35$ & 0.982 \\
CACMPDA & $\mathrm{Y}=0.503 \mathrm{x}-14.01$ & 0.993 & $\mathrm{Y}=0.549 \mathrm{x}-17.40$ & 0.987 \\
PDA & $\mathrm{Y}=0.376 \mathrm{x}-10.99$ & 0.994 & $\mathrm{Y}=0.470 \mathrm{x}-16.84$ & 0.979 \\
\hline
\end{tabular}


hojas de palma de durazno. Este comportamiento permite entender claramente que la velocidad de crecimiento de estos microorganismos depende de las características del medio y de la capacidad de adaptación de los hongos, a las diferentes fuentes de carbono, en el proceso de biodegradación de residuos lignocelulosicos.

\section{Conclusiones}

$\mathrm{L}$ a cepa de $P$. ostreatus y el medio PDA preparado con solución de cascarilla de arroz, mostró la mayor velocidad de crecimiento radial y producción de biomasa fúngica, esto demuestra que este residuo lignocelulosico tiene un alto potencial, para la aplicación de fermentación en medio solido del Pleurotus spp., sea para obtención de biomasa o producción de setas comestibles.

\section{Agradecimiento}

A la Universidad Técnica Estatal de Quevedo por el financiamiento, y a la Universidad Autónoma Chapingo y Universidad Autónoma del Estado de Hidalgo de los Estados Unidos Mexicanos, por su participación como contraparte Internacional para el desarrollo del proyecto de investigación: Transformación de Residuos y Residuales Agrícolas y Agroindustriales mediante métodos físicos, químicos y biológicos en alimento para uso animal.

\section{Bibliografía}

Bernardi, E., Minotto, E., \& Soares do Nascimento, J. (2013). Evaluation of growth and production of Pleurotus sp. in sterilized substrates. Agricultural Microbiology / Scientific Article, 80(3), 318-324.

Cruz, A., Guaman, M., Castillo, M., Glorio, P., \& Martinez, R. (2015). Fibra dietaria en subproducto de mango, maracuya, guayaba y palmito. R Politécnica, 36(2).

Cuesta, M., \& Muñoz, R. (2010). Extraccion de pectina a partir de la corteza de maracuya (Passiflora edulis var. Flavicarpa degener). Revista Politécnica, 31, 91-96.

Gaitan-Hernandez, R., \& Silva-Huerta, A. (2016). Aprovechamiento de residuos agrícolas locales para la producción de Pleurotus spp. en una comunidad rural de
Veracruz, México. R Mexicana de Micología, 43, 43-47. Martinez, D., Buglione, M., Filippi, M., Reynoso, L., Rodriguez, G., \& Aguero, M. (2015). Evaluación del crecimiento micelial de Pleurotus ostreatus y Agrocybe aegerita sobre orujos de pera. Anales de Biología, 37, $1-10$.

Ortega, G., Bueno, G., Betancourt, D., Alvarez, I., \& Gonzales, A. (2005). Biotransformacion de Residuos Lignocelulosicos con Hongos Pleurotus. Revista CENIC Ciencias Biológicas, 36.

Pelaz-Acero, A., Meneses-Mayo, M., Miranda-Romero, A., Ayala-Martinez, M., Crosby-Galvan, M., Loera-Corral, O., \& Megias-Rivas, D. (2011). Enzimas fibrolíticas producidas por fermentación en estado sólido para mejorar los ensilajes de caña de azúcar. Agrociencia, 45, 675-685.

Pineda-Insuasti, J., Ramos-Sanchez, L., \& Soto-Arroyave, C. (2013). Cinética del crecimiento de Pleurotus ostreatus en la etapa de producción del cuerpo fructífero. Revista ICIDCA sobre los derivados de la caña de azúcar, 47(3), 56-61.

Rios, MD., Hoyos, JL., \& Mosquera, SA. (2010). Evaluacion de los párametros productivos de la semilla de Pleurotus ostreatus. Propagada en diferentes medios de Cultivo. Facultad de Ciencias Agropecuarias, 8(2), 86-94.

Rojas, A., \& Hormaza, A. (2014). Evaluación del crecimiento y compatibilidad de hongos de la podredumbre blanca. Revista Ciencia en Desarrollo, 5(2), 197-205.

Sanchez, A., Esqueda, M., Gaitan-Hernandez, R., Cordova, A., Coronado, M. (2008). Uso potencial del rastrojo de tomate como sustrato para el cultivo de Pleurotus spp. Revista Mexicana de Micologia, 28, 17-24.

Sanchez-Santillan, P., Meneses-Mayo, M., Miranda-Romero, L., Santellano-Estrada, E., Alarcon-Zuniga, B. (2015). Fribrinolytic activity and gas production by Pleurotus ostreatus-IE8 and Fomes fomentarius-EUM1 in bagasse cane. Rev. MVZ Córdoba, 20, 4907-4916.

Suarez, C., \& Holguin, M. (2011). Evaluación de medios de cultivo sintéticos y cereales para la producción de semilla de setas comestibles. Revista Colombiana de Ciencias Hortícol, 5(1), 130-140.

Valverde, A., Sarria, B., \& Monteagudo, J. (2007). Análisis comparativo de las características fisicoquímicas de la cascarilla de arroz. Scientia et Technic, 37, 255-260.

Wisbeck, E., Alves, E., Gonçalves de Lima, S., Miranda Gern, R., Lange Silveira, M., \& Aparecida Furlan, S. (2016). Maintenance culture medium and inoculum based on peach palm leaves for Pleurotus spp. production. Agricultural Microbiology / Scientific Article, 83, 1-7. 